\title{
El arte como instrumento para comprender la diferencia entre el enfermo y la enfermedad
}

\author{
Art as an instrument to grasp the difference between the ill and the illness
}

En este artículo continuamos destacando la importancia del arte en la enseñanza de grado y en los profesionales en formación. Consideramos necesario enfatizar la capacidad que el lenguaje simbólico del arte tiene para reflejar la dimensión inefable humana. ${ }^{1}$

En el presente artículo analizaremos cómo el arte puede ayudar a comprender la diferencia existente entre el proceso fisiopatológico que afecta a un individuo (el enfermo) y la construcción teórica que se elabora para su abordaje interpretativo (la enfermedad).

En este sentido resulta fundamental señalar que los nombres que la medicina utiliza para designar a las distintas enfermedades constituyen rótulos arbitrarios, aunque validados por su utilidad práctica y por el consenso; estos logran segmentar, en porciones humanamente entendibles y manejables, aquello que en realidad es una inmensa y continua red de imbricados procesos fisiopatológicos.

Este artilugio lingüístico le ha permitido a generaciones de médicos poder pensar en estos problemas en términos de enfermedades y procurar, a partir de su conceptualización, resolverlos o al menos mitigarlos. ${ }^{2}$ Este hecho explica por qué existen enfermos que presentan cuadros clínicos "atípicos", como por ejemplo, una neumonía sin fiebre o un infarto de miocardio sin dolor anginoso. Confundir entonces un nombre teórico (enfermedad) con lo que en realidad le sucede al paciente (fisiopatología), es confundir las palabras con las cosas (Foucault), o dicho de otra forma, creer erróneamente que el mapa es el territorio que éste intenta simplemente representar, confusión que conlleva el riesgo de no poder llegar a diagnósticos clínicos complejos.

Desde el arte, existe un ejemplo esclarecedor de este punto de vista, en la pintura "Las Meninas" de Diego Velázquez. En esta obra el artista se retrató a sí mismo pintando a la infanta Margarita con su séquito (Las Meninas) en la corte del rey español Felipe IV. Sin embargo, la misma escena ha sido recreada de forma muy distinta por otros autores: Pablo Picasso (pintura), Salvador Dalí (pintura), Manolo Valdés (escultura) y
Michel Foucault (análisis filosófico). Todos ellos representaron un mapa distinto de una misma realidad, y pudieron hacerlo porque la riqueza de la realidad es inagotable en comparación con los estrechos límites de su representación. ${ }^{4}$

Este fenómeno explica también por qué el avance en el conocimiento de un determinado proceso patológico o su modificación evolutiva a raíz del tratamiento implementado, motiva la creación de nuevos nombres y clasificaciones médicas. Los antiguos rótulos resultan ahora insuficientes, requiriéndose una expansión y enriquecimiento de la terminología heredada, a fin de contribuir a generar una mirada médica más precisa, en consonancia con la forma actual de sanar y enfermar.

Nuevamente desde el arte tenemos ejemplos esclarecedores de este fenómeno tales como en las obras de Pablo Picasso (cubismo), Oskar Kokoschka (expresionismo), Lewis Caroll (Jabberwocky) y James Joyce (Finnegans Wake), todos ellos autores que crearon nuevos términos (pictóricos y literarios, respectivamente), a fin de expandir sus fronteras conceptuales. ${ }^{5-7}$

Por ejemplo, la obra de James Joyce "Finnegans wake". Si bien está escrita básicamente en inglés, Joyce emplea en ella palabras de por lo menos 70 idiomas, además de un sinnúmero de neologismos del tipo portmanteaux, es decir, palabras que nacen de la fusión de otras, con el objetivo de romper la linealidad del pensamiento formal y crear nuevos conceptos.

Ejemplo en el texto: ${ }^{8}$

Riverrun = river (inglés) + run (inglés): expresa sintéticamente "el río que corre".

Regginbrow = rainbow (inglés) - reggen (alemán) + eyebrow (inglés): expresa sintéticamente "el arco iris es un ojo en la cara de la lluvia".

Concluimos entonces que la incorporación del arte como instrumento educativo en la medicina contribuye a poder lograr una mayor comprensión respecto de la profunda diferencia existente entre los conceptos de enfermo (la realidad) y de enfermedad (su representación). 
Dr. Carlos G. Musso y Dra. Paula A. Enz

Escuela de Medicina, Instituto Universitario,

Hospital Italiano de Buenos Aires,

Buenos Aires, Argentina

http:/ /dx.doi.org/10.5546/aap.2015.292

\section{REFERENCIAS}

1. Musso CG, Enz PA. El arte como instrumento educativo en medicina. Arch Argent Pediatr 2014;112(6):494-5.

2. Musso CG. Obras maestras del arte universal y la medicina: Muerta mimosa tuya quiero ser Elena Bellamuerte de Macedonio Fernández (1874-1952). Evid Act Pract Ambul.En prensa 2015.
3. Musso CG, Enz PA. Semiótica médica. Principios lingüísticos y comunicacionales de uso médico. Buenos Aires: Del hospital ediciones; 2007.

4. Musso CG. Diego Velászquez and his painting: The triadic matter of reality. Humane Medicine Health Care 2007;7(1).

5. Musso CG. We need new terms to better explore emergent clinical settings. Commentary. [Acceso: 1 de abril de 2015]. Disponible en: http:/ / www.cardiab.com/ content/12/1/156/comments\#1978698.

6. MussoCG. Obras maestras del arte universal y la medicina: Autorretrato de Oskar Kokoschka (1886-1980). Evid Act Pract Ambul. En prensa 2015.

7. Musso CG, Enz PA. Arte y naturaleza humana XIII. Rev Hosp Ital B Aires 2013; 33(3):109-10.

8. Joyce J. Finnegans wake. New York: Penguin; 1976.

\section{La experiencia clínica y el valor de una publicación científica Clinical experience and the value of scientific publications}

En el curso de mi carrera profesional he oído las más variadas opiniones de colegas sobre la importancia de publicar artículos científicos; desde opiniones exageradamente elogiosas de esa actividad, hasta las más desvalorizantes. Entre estas últimas, pueden citarse las siguientes:

1. Hay gente que no publica pero que "trabaja muy duro", ve muchos pacientes y eso es lo que vale.

2. La experiencia se logra con el trabajo.

3. Publicar significa muy poco.

A estos argumentos podemos oponer las siguientes reflexiones:

1. Las personas que publican artículos científicos también "trabajan muy duro", solo que, además, le roban tiempo a la vida familiar, al descanso, a las distracciones, y a otras actividades para poder hacer ese trabajo extra.

2. No es verdad que la experiencia se logra con el trabajo. El trabajo es condición necesaria pero no suficiente. En el caso de la clínica pediátrica (como en el de muchas otras áreas), la experiencia no es "haber visto muchos pacientes", sino haber reflexionado sobre los pacientes que se han visto. Y... ¿en qué consiste esa reflexión?, consiste en poder juntar toda la información sobre los pacientes que se han visto, evaluar su confiabilidad, ordenarla, agruparla, procesarla, analizarla, ponerla en un contexto ${ }^{1} \mathrm{y}$ finalmente sacar una conclusión válida, que sintetiza la "experiencia".

Pero para poder hacer todo esto hay que disponer de la información adecuadamente registrada. Muchos clínicos podemos vanagloriarnos de la "enorme cantidad de anginas" que hemos visto en la vida profesional, y la "extraordinaria experiencia" que eso significa. Pero el valor de esta experiencia se desvanece rápidamente cuando uno pregunta si hemos registrado con cuidado en todos estos años las características clínicas de las "anginas" atendidas, si habíamos hecho una encuesta sistemática sobre la evolución de las mismas (a los quince días, por ejemplo), si habíamos evaluado el grado de cumplimiento de las indicaciones prescriptas, etc. Sin esa información, ninguna reflexión puede hacerse sobre esos 30 años de haber "visto" anginas.

Es frecuente en los congresos médicos, asistir a presentaciones sobre pacientes con una determinada patología y volver entusiasmado para revisar la propia casuística porque "en el hospital tenemos muchos más pacientes de este tipo que ellos". Sin embargo, cuando volvemos al hospital y revisamos las historias clínicas, nos damos cuenta que, efectivamente, tenemos más pacientes que "ellos", pero no los podemos analizar porque a uno le falta una radiografía, al otro un análisis, el otro hace cuatro años que no consulta y nadie lo llamó por teléfono, etc. Para reflexionar sobre lo que se hizo también es necesario disponer de un registro sistemático de lo que se hizo. En mi opinión todo el seguimiento de pacientes crónicos debería ser hecho bajo un protocolo determinado para poder así evaluarlo en 
el tiempo. En caso contrario es muy difícil adquirir "experiencia", reflexionar sobre lo que se hizo sobre datos concretos, y así saber si estamos haciendo las cosas bien. Si esta reflexión está bien hecha, por más modesta que sea, entonces merece ser compartida con otros colegas, ya que finalmente de eso se trata una publicación.

3. Publicar un artículo significa muchas cosas. ${ }^{2}$ Significa por ejemplo, haber sido capaz de registrar la información, de disponer de información válida, de ordenarla, y de esforzarse en extraer resultados. Significa haber leído la bibliografía, porque no se puede escribir algo con la petulancia de ignorar lo que otros han hecho en el mismo tema, aunque los otros sean nuestra "competencia" científica. Significa también poner nuestras ideas en un estructura especial, ${ }^{2,4}$ y con la palabra escrita, y esto es una habilidad que hay que desarrollar. Se puede ser un gran clínico sin ser capaz de poder escribir bien un artículo, porque son dos destrezas diferentes. Indagar en la palabra no es tarea fácil, ${ }^{5}$ es algo que nadie nos enseña, pero si enriquecemos nuestro lenguaje, enriquecemos nuestro pensamiento y nuestra capacidad de comunicarnos. Como toda destreza, la escritura se puede aprender. Escribir un artículo científico también quiere decir que uno se va a exponer a la crítica de los pares, y que está dispuesto a aceptarla y a cambiar el texto. Rara vez las revistas aceptan un artículo sin cambios, la mayoría de las veces se devuelve con muchas observaciones, preguntas, y sugerencias. Someterse a esas críticas es una exposición que exige cierta madurez científica, y un hábito cultural de aceptar críticas. Hay una segunda exposición que es cuando el artículo es finalmente publicado, momento en el cual nuestros pares pueden volver a criticarlo, con mayor o menor virulencia. Esto es verdad siempre que en el artículo incluyamos nuestro pensamiento, nuestras propias ideas, porque hay autores que, como no se animan a poner lo que piensan, copian las ideas de otros, ciencia sin seso, como lo ha descripto magistralmente Cereijido. ${ }^{6}$

Todo esto se puede encontrar detrás de una publicación científica. Se puede ser un muy buen pediatra sin gozar de la habilidad de escribir bien, pero publicar un artículo es una aventura intelectual que recomiendo hacer al menos una vez en la vida. No dude el lector que si lo logra, tendrá la extraordinaria sensación de haber agregado una partícula más al saber universal, de haber puesto en este mundo algo propio que antes no estaba. Se trata de una tarea creativa, basada en la "experiencia", es decir en la reflexión de lo que hacemos, lo cual sin duda, tiene mucho valor en sí mismo y que además, podemos compartirla con nuestros pares.

$$
\begin{array}{r}
\text { Prof. Dr. Horacio Lejarraga } \\
\text { Universidad de Buenos Aires } \\
\text { Autoridad de Cuenca Matanza Riachuelo }
\end{array}
$$

(ACUMAR)

http:/ /dx.doi.org/10.5546/aap.2015.293

\section{REFERENCIAS}

1. Bazerman C. Shaping written knowledge. The genre and activity of the experimental article in science. Madison: University of Winsconsin Press; 1988.

2. Lejarraga H. La escritura de un artículo científico. Arch Argent Pediatr 2001;99(3):273-80.

3. Albert T. The A-Z of medical writing. London: BMJ Books; 2000.

4. Day RA. Cómo escribir y publicar trabajos científicos. $2^{\text {da }}$ ed. Washington DC: Organización Panamericana de la Salud; 1996.

5. Borges JL. El idioma de los argentinos. Buenos Aires: Seix Barral; 1994. Capítulo 1: Indagación de la palabra; 11-25.

6. Cereijido M. Ciencia sin seso, locura doble. México: Siglo Veintiuno; 1994. 